\title{
Undecidability of Static Analysis
}

\author{
WILLIAM LANDI \\ Siemens Corporate Research Inc.
}

\begin{abstract}
Static analysis of programs is indispensable to any software tool, environment, or system that requires compile-time information about the semantics of programs. With the emergence of languages like $\mathrm{C}$ and LISP, static analysis of programs with dynamic storage and recursive data structures has become a field of active research. Such analysis is difficult, and the static-analysis community has recognized the need for simplifying assumptions and approximate solutions. However, even under the common simplifying assumptions, such analyses are harder than previously recognized. Two fundamental static-analysis problems are may alias and must alias. The former is not recursive (is undecidable), and the latter is not recursively enumerable (is uncomputable), even when all paths are executable in the program being analyzed for languages with if statements, loops, dynamic storage, and recursive data structures.
\end{abstract}

Categories and Subject Descriptors: D.3.4 [Programming Languages]: Processors; F.1.1 [Computation by Abstract Devices]: Models of Computation-bounded-action devices; F.4.1 [Math Logic and Formal Languages]: Mathematical Logic-computability theory

General Terms: Languages, Theory

Additional Key Words and Phrases: Abstract interpretation, alias analysis, data flow analysis, halting problem, static analysis

\section{INTRODUCTION}

Static analysis is the processes of extracting semantic information about a program at compile time. One classical example is the live-variables [Hecht 1977] problem; a variable $x$ is live at a statement $s$ iff on some execution $x$ is used (accessed) after $s$ is executed without being redefined. Other classical problems include reaching definitions, available expressions, and very busy expressions [Hecht 1977]. There are two main frameworks for doing static analysis: data flow analysis [Hecht 1977] and abstract interpretation [Cousot and Cousot 1977]. The framework is not relevant to this paper, since we show that two fundamental static-analysis problems are harder than previously acknowledged, regardless of the framework used.

We view the solution to a static-analysis problem as the set of "facts" that hold for a given program. Thus, for live variables the solution is $\{(x, s) \mid$ variable $x$ is live at statement $s$. With that in mind, we review a few

Author's address: Siemens Corporate Research Inc., 755 College Road East, Princeton, NJ 08540; email: wlandi@scr.siemens.com.

Permission to copy without fee all or part of this material is granted provided that the copies are not made or distributed for direct commercial advantage, the ACM copyright notice and the title of the publication and its date appear, and notice is given that copying is by permission of the Association for Computing Machinexy. To copy otherwise, or to republish, requires a fee and/or specific permission.

(c) $1992 \mathrm{ACM} 1057-4514 / 92 / 1200-0323 \$ 01.50$

ACM Letters on Programming Languages and Systems, Vol. 1, No. 4, December 1992, Pages 323-337. 
definitions:

-A set is recursive iff it can be accepted by a Turing machine that halts on all inputs.

-A set is recursively enumerable iff it can be accepted by a Turing machine which may or may not halt on all inputs.

Static analysis originally concentrated on Fortran and was predominately confined to a single procedure (intraprocedural analysis) [Kam and Ullman 1976; Kildall 1973; Ullman 1973l. However, even this simple form of static analysis is not recursive. The difficulty lies in conditionals. There are, in general, many paths through a procedure, but not all paths correspond to an execution. For example, consider the following:

$$
\begin{array}{ll}
\text { if }(x>-1) & y=1 \\
\text { if }(x<0) & y=-1
\end{array}
$$

Execution of this fragment always executes exactly one then branch. It is impossible for both or neither then branches to be executed. Static analysis is not recursive, since determining which paths are executable is not recursive. To overcome this problem, static analysis is performed assuming that all paths through the program are executable [Aho et al. 1986]. This assumption is not always valid, but it is safe. ${ }^{1}$ It also simplifies the problem and allows static analysis of Fortran procedures to be done fairly efficiently. Some approaches (for example [Wegman and Zadeck 1991]) categorize some paths as being not executable. However, these techniques have limited applicability, and must often assume that paths are executable.

With a basis of a firm understanding of intraprocedural static analysis of Fortran, static analysis of entire programs (interprocedural analysis) was investigated. Myers [1981] came up with the negative result that many interprocedural static-analysis problems are $N P$ complete. Practically this means that interprocedural static analysis must make further approximations over intraprocedural analysis or take an exponential amount of time.

With the emergence of popular languages like $C$ and Lisp, the staticanalysis community has turned its attention to languages with pointers, dynamic storage, and recursive data structures. It is widely accepted that static analysis under these conditions is hard. The general feeling is that it is probably NP complete [Landi and Ryder 1991; Larus 1989; Larus and Milfinger 1988]; this is incorrect. Recently, the problem of finding aliases was shown to be P-space hard [Landi 1992]. Unfortunately, this is still an underestimate.

An alias occurs at some point during execution of a program when two or more names exist for the same storage location. For example, the $\mathrm{C}$ statement " $p=\& v$ " creates an alias between * $p$ and $v$. Aliases are associated with program points, indicating not only that $* p$ and $v$ refer to the same location

${ }^{1}$ The term conservative is used in Aho et al. [1986] instead of safe.

ACM Letters on Programming Languages and Systems, Vol. 1, No. 4, December 1992. 
during execution, but also where in the program they refer to the same location. Aliasing, statically finding aliases, is a fundamental problem of static analysis. Consider the problem of finding live variables for

$$
\begin{array}{ll}
s_{1}: & v=1 \\
s_{2}: & p=\& v \\
s_{3}: & w=2 ; \\
s_{4}: & \text { printf(“\%d”, } \left.{ }^{*} p\right) ;
\end{array}
$$

The variable $v$ is live at $s_{3}$ only because ${ }^{*} \mathrm{p}$ is aliased to $\mathrm{v}$ when program point $s_{4}$ is executed. Aliasing also influences most interesting static-analysis problems. Any problem that is influenced by aliasing is at least as hard as aliasing. There are two types of aliasing:

- May alias: Find the aliases that occur during some execution of the program.

- Must alias: Find the aliases that occur on all executions of the program.

Finding the aliases can mean determining the set of all aliases which hold at some associated program points, or determining whether $x$ and $y$ are names for the same location at a particular program point $s$. We use the latter meaning, since, in general, the set of all aliases may be infinite in size. We formally define may alias as a Boolean function:

may-alias $_{P}(s,\langle x, y\rangle)$ is true iff there is an execution of program $P$ to program point $s$ (including the effects of executing $s$ ) on which $x$ and $y$ refer to the same location.

Must alias is defined analogously. We show that, for languages with if statements, loops, dynamic storage, and recursive data structures, intraprocedural may alias is not recursive (i.e., is undecidable) and that intraprocedural must alias is not recursively enumerable (i.e., is uncomputable) even when all paths in a program are executable by reducing [Hopcroft and Ullman 1979, pp. 321-322] the halting problem into an alias problem. This is different from the result of Kam and Ullman [1977] that the MOP solution is undecidable for monotone frameworks.

\section{REDUCTION OF THE HALTING PROBLEM TO AN ALIAS PROBLEM}

A Deterministic Turing Machine (DTM) [Aho et al. 1976] is a tuple $\left(\mathrm{Q}, \mathrm{T}, \mathrm{I}, \delta, \beta, \mathrm{q}_{0}, \mathrm{q}_{f}\right)$ where:

$-\mathrm{Q}=\left\{\mathrm{q}_{1}, \mathrm{q}_{2}, \ldots, \mathrm{q}_{n_{Q}}\right\}$ is the set of states,

$-\mathrm{T}=\left\{\sigma_{1}, \sigma_{2}, \ldots, \sigma_{n_{T}}\right\}$ is the set of tape symbols,

$-\mathrm{I} \subset \mathrm{T}$ is the set of input symbols,

$-\delta:(\mathrm{Q} \times \mathrm{T}) \rightarrow(\mathrm{Q} \times \mathrm{T} \times\{\mathrm{L}, \mathrm{R}, \mathrm{S}\})$ is the transition function, ${ }^{2}$

$-\beta \in \mathrm{T}-\mathrm{I}$ is the blank symbol,

\footnotetext{
${ }^{2} \mathrm{~L}$ moves tape head left; $\mathrm{R}$ moves tape head right; and $\mathrm{S}$ leaves the tape head where it is.
} 
$-\mathrm{q}_{0} \in \mathrm{Q}$ is the start state,

$-\mathrm{q}_{f} \in \mathrm{Q}$ is the final state.

We assume that $\delta$ is a total function and that the DTM will not move off the left end of the tape. In general, neither of these assumptions is true, but any Turing machine can be modified to conform to them.

In this section, we specify a machine reduce (Figure 1) which takes a DTM $M$ and input string $w$ and procedures a program $C$ such that

- may-alias $(s,\langle * *$ current_state, valid_simulation $\rangle)$ is true iff $M$ halts on w.

- must-alias $_{C}(s,\langle * *$ current_state, not_valid $\rangle)$ is true iff $M$ does not halt on w.

-all paths through $\mathrm{C}$ are executable.

\subsection{Representing an ID}

An Instantaneous Description (ID) is an encoding of the following information:

- contents of the DTM's tape

-current state of the DTM

- location of the tape head.

An ID is usually represented by a string $x q_{z} y \in \mathrm{T}^{*} \mathrm{QT}^{*}$ where the tape contains $x y$ infinitely padded to the right with blanks; the current state is $q_{i}$; and the tape head scans the first character of $y .{ }^{3}$ We encode this information in the alias pattern of a program execution, by which we mean the relationship of names to each other.

We use a doubly-linked list to represent the tape of a DTM:

\begin{tabular}{|l|l|l|}
\hline prev & sym & next \\
\hline
\end{tabular}

For each $\sigma_{\imath} \in \mathrm{T}$ we create a variable $\sigma_{i}$. The "sym" field points to $\sigma_{i}$ iff the tape location contains $\sigma_{2}$. Thus the tape that contained "hello" padded to the right with blanks $(\beta)$ is represented by the alias pattern:

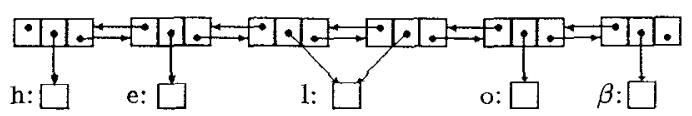

For each $\mathbf{q}_{l} \in \mathbf{Q}$ we create a variable $\mathbf{q}_{l}$, and there are two additional variables, current_state and tape_head. Current_state points to the current state of the machine, and tape_head indicates the tape-head location by pointing into the list representing the tape. The $\mathrm{ID}=h e q_{2} l l o$ is

${ }^{3} q_{l}$ is underlined in $x q_{2} y$ to make the state stand out from the tape string.

ACM Letters on Programming Languages and Systems, Vol. 1, No. 4, December 1992. 


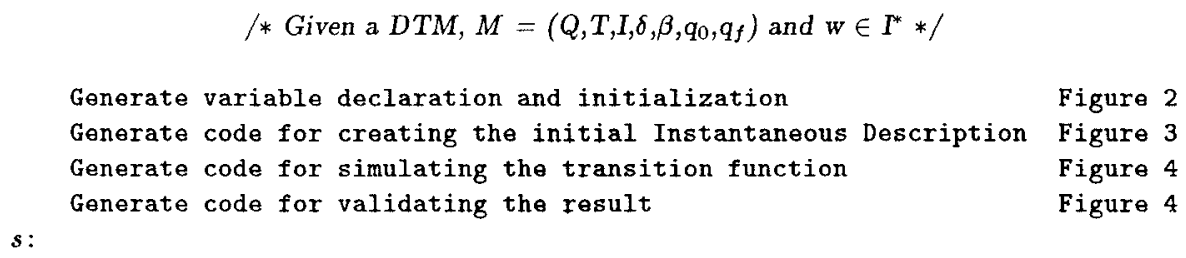

Fig. 1. Outline of the machine reduce.

represented by
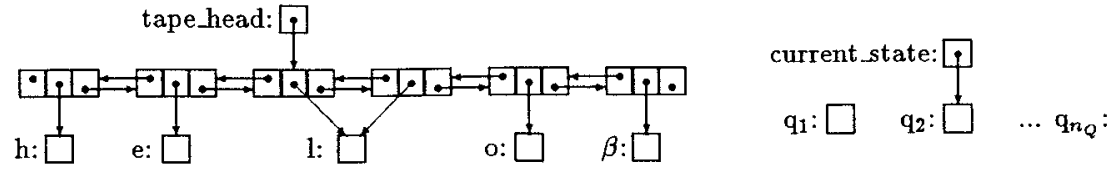

\subsection{Programming Language}

In order to perform the required reduction, we need to construct a program from a DTM. The program is in $\mathrm{C}$, but it could be in any language with if statements, loops, dynamic storage, and recursive data structures. We use the address operator $(\&)$, but it is not fundamentally necessary to the proof. To specify a C program from a DTM, we need the metastatements: \#for and \#if. The syntax and meaning of these are relatively straightforward and should be apparent from the following examples:

\begin{tabular}{|c|c|}
\hline $\begin{array}{c}\text { \#for } i=1 \text { to } 3 \\
x_{1}=i \\
\text { \#endfor }\end{array}$ & represents \\
\hline $\begin{array}{c}\text { \#for } i=1 \text { to } 3 \\
x_{1}=i \\
\# \text { if } i \text { is odd } \\
y_{1}=i \\
\text { \#endif } \\
\text { \#endfor }\end{array}$ & represents \\
\hline
\end{tabular}

We use next_bool for reading program input. It returns the next Boolean value from the input stream. If the end of the stream has been encountered, it returns 0 .

\subsection{Simulating a DTM}

In Section 2.1 we showed how we represent an ID with aliases. In this section we show how to simulate a DTM with the alias pattern of executions of a particular program. We now specify reduce (Figure 1) which constructs a program from a DTM $M=\left(\mathrm{Q}, \mathrm{T}, \mathrm{I}, \delta, \beta, \mathrm{q}_{0}, \mathrm{q}_{f}\right)$ with initial input $\mathrm{w} \in \mathrm{I}^{*}$ such that $* *$ current_state is aliased to valid_simulation on some execution to program point $s$ iff machine $M$ halts on input $\mathrm{w}=x_{1} x_{2} \ldots x_{n_{w}}$. 
LEMMA 2.1. The code generated by Figure 3 creates the data structure that represents the initial configuration of $M\left(q_{0} x_{1} x_{2} \ldots x_{n_{w}}\right)$ in the manner described in Section 2.1.

Proof. This is evident from inspection of the code; "current_state $=\& q_{0}$;" points current_state to $q_{0}$; tape_head points to the first element of the linked list which corresponds to the tape head of $M$ being on the beginning of the tape. Finally, NEXT_SYM $(i)$ points the "sym" field of the $i$ th element of the linked list to the variable representing the $i$ th symbol on the tape $\left(x_{i}\right)$. This is exactly what is required by Section 2.1 for the initial ID $\underline{q}_{0} x_{1} x_{2} \ldots x_{n_{w}}$.

As an example, consider the case where $\mathrm{T}=\{\mathrm{h}, \mathrm{e}, \mathrm{l}, \mathrm{o}, \beta\}, \mathrm{w}=$ "hello," and $\mathrm{q}_{0}=\mathrm{q}_{2}$. The initial ID is $q_{2}$ hello, and the code generated by Figure 3 produces:
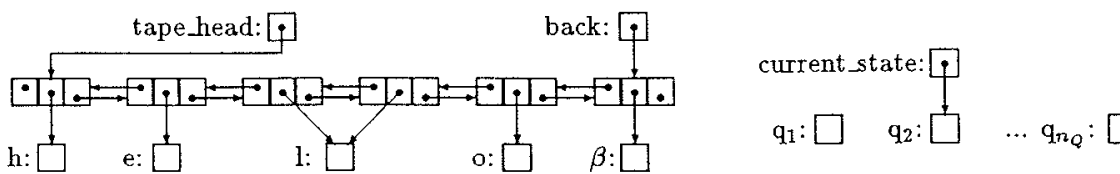

Notice that back points to the end of the linked list representing the tape. This allows us to add a new tape element to the end of the tape and, as will be seen later, is used to ensure that we never run off the right end of the tape.

The declaration and initialization of the variables of the program used to simulate a DTM are specified in Figure 2. All the variables except yes, no, not_valid, and valid_simulation have already been explained in Section 2.1. Three of the four new variables are simply to record whether the current path is a valid simulation of $M$. Yes points to valid simulation iff the current path is a simulation of $M$; yes points to not_valid otherwise. The fourth variable (no) is just a "don't care" location for pointers that must refer to something other than yes. No always points to not_valid. Continuing our example, the initialization specified in Figure 2 followed by the code specified in Figure 3 for $T=\{h, e, l, o, \beta\}, w=$ "hello," and $q_{0}=q_{2}$, produces:

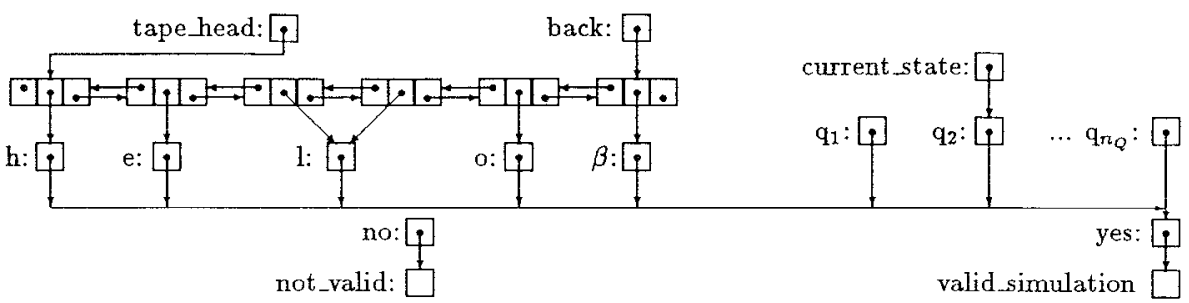

The remainder of the program created by reduce is mostly a while loop (Figure 4). Each pass through the body of the loop represents one application of the transition function $(\delta)$. The first part of the loop (ADD_TO_END) simply adds a new tape location, initially blank, to the right end of the tape. This ensures that whenever the simulated DTM moves right on the tape, a tape location is available to it. The remainder of the loop is a nested 


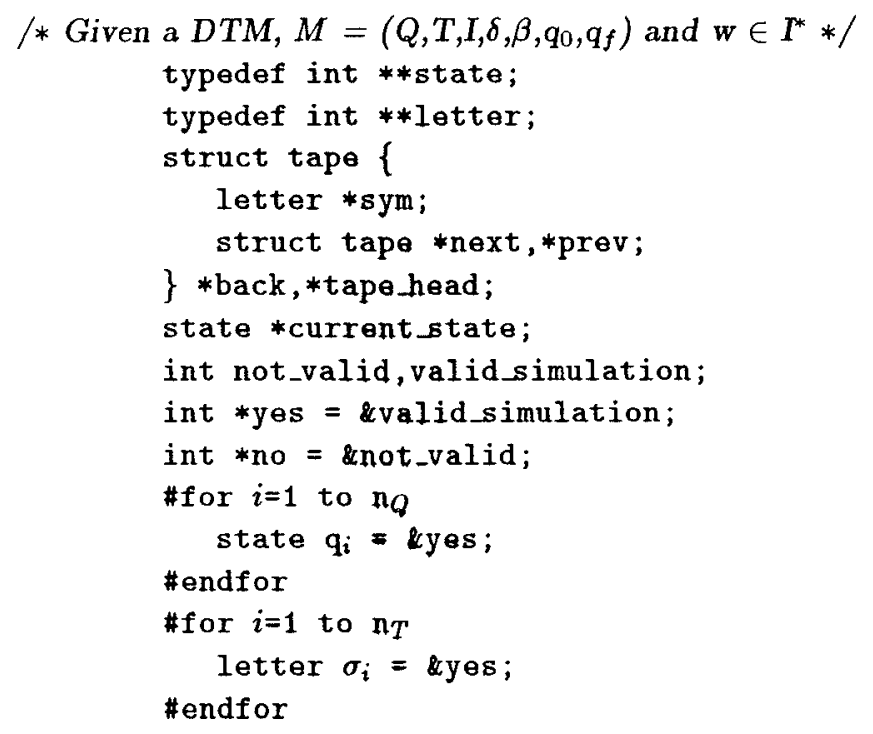

Fig. 2. Variable declaration and initialization.

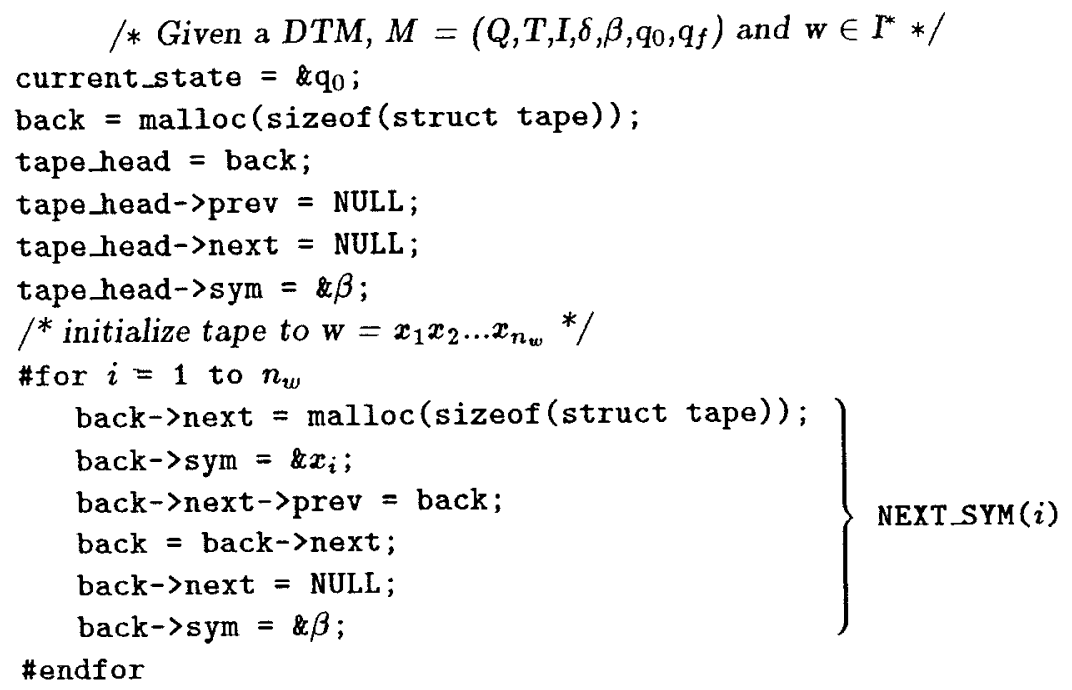

Fig. 3. Initial Instantaneous Description (ID). 


$$
\text { /* Given a DTM, } M=\left(Q, T, I, \delta, \beta, q_{0}, q_{f}\right) \text { and } w \in I^{*} * /
$$

/* next_bool returns the next boolean value from the input stream. */ back $\rightarrow$ next $=$ malloc $($ sizeof (struct tape) $)$;

Fig. 4. Representation of the transition function. 
if-then-else-if

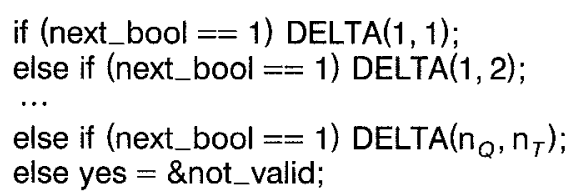

where DELTA(i,j) (explained below) is the code to implement $\delta\left(\mathrm{q}_{\imath}, \sigma_{j}\right)$. The code

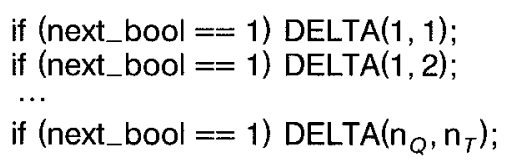

would also be valid and does not require an artificially deep if-then-else-if nesting. However, in the former, a pass through the loop either is not a valid simulation or represents exactly one transition of the DTM $M$. In the latter, a pass through the loop is an invalid simulation or represents a legal sequence of 0 to $\mathrm{n}_{Q} * \mathrm{n}_{T}$ transitions of $M$. The former is preferable because it makes the proof of correctness easier.

LEMMA 2.2. If yes points to not_valid before the loop specified in Figure 4 is executed then yes still points to not_valid after execution of the loop.

Proof. Inspection of the code reveals that in the while loop only \&not_valid can be assigned to yes.

The next lemma basically states that if the execution is a valid simulation of $M$ before executing the loop generated by Figure 4, then one path through the loop simulates the next transition of $M$, and all other paths are not valid simulations.

LEMMA 2.3. If before the loop specified in Figure 4 is executed, yes points to valid_simulation; the ID encoded by the alias pattern is $x q_{i} y$; and $x q_{u} y \vdash_{M} x^{\prime} q_{j} y^{\prime}$, then on all but one path through the loop yes points to not_valid; and on the remaining path, yes points to valid_simulation, and the alias pattern represents the ID $x^{\prime} \underline{q}_{y} y^{\prime}$.

Proof. We illustrate this proof with the example heq $q_{2} l l o \vdash_{M} \mathrm{hq}_{1}$ eelo (therefore $\left.\delta\left(q_{2}, l\right)=\left(q_{1}, e, L\right)\right)$. For this example, the alias pattern before execution of the loop is

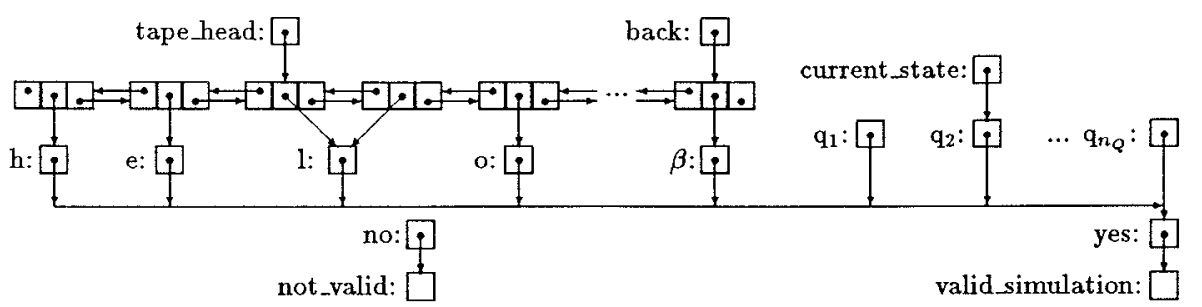

ACM Letters on Programming Languages and Systems, Vol. 1, No. 4, December 1992. 
Before execution of the while loop, all $\sigma \in \mathrm{T}$, and all $\mathbf{q} \in \mathrm{Q}$ point to yes. ${ }^{4}$ The first part of the loop, as stated earlier, just expands the tape. The last else clause simply points yes to not_valid signaling an invalid simulation. Now consider the code for DELTA(i,j) which represents the application of $\delta\left(q_{i}, \sigma_{j}\right)$. Notice that we can only use this rule if $M$ is in state $\mathrm{q}_{\imath}$ and if the tape head is reading $\sigma_{y}$. Thus we would like to say (in pseudo-C)

$$
\begin{aligned}
& \text { if }\left(* \text { current_state } \neq \mathbf{q}_{i} \text { or } * \text { tape_head }->\operatorname{sym} \neq \sigma_{j}\right) \\
& \text { yes }=\text { \&not_valid } / * i . e ., \text { not a valid simulation } * /
\end{aligned}
$$

but this would create paths which are not executable in the program. The first four statements of DELTA $(i, j)$ do exactly (1) without using a conditional. The statements " $\mathrm{q}_{t}=\&$ no; $\sigma_{l}=\&$ no" point $\mathbf{q}_{l}$ and $\sigma_{J}$ to no. All other states and alphabet symbols still point to yes. In our running example (where $\mathrm{q}_{\imath}=\mathrm{q}_{2}$ and $\sigma_{j}=1$ ) we have

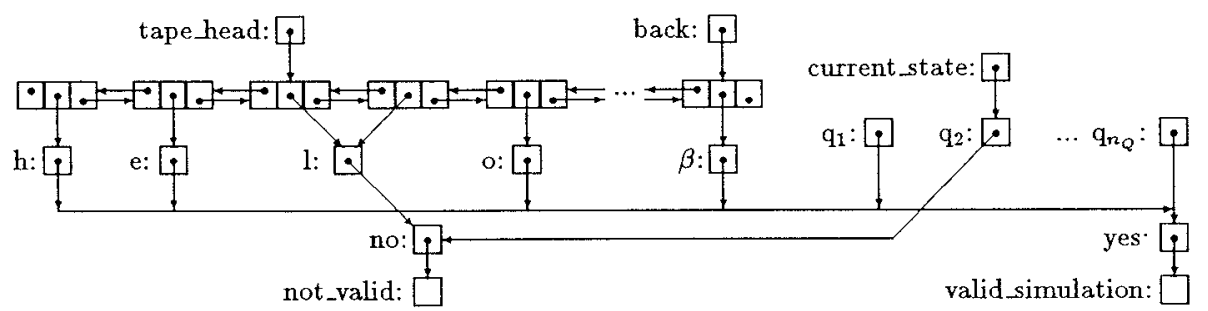

The statements, "** current_state $=$ \&not_valid" and "***(tape_head $->$ sym $)$ $=\&$ not_valid" make sure that the application of $\delta\left(q_{i}, \sigma_{j}\right)$ is applicable. Two cases that can occur:

- The tape head is scanning $\sigma_{j}(\mathrm{l})$, and $M$ is in state $q_{1}\left(\mathrm{q}_{2}\right)$

This means that $* *$ current_state is no, and $* *$ (tape_head $->$ sym) is also no. Thus both of these statements effectively are "no = \&not_valid," and the value of yes is unchanged and still points to valid_simulation. This is the path through the loop that is a valid simulation of $M$. For all $i, j$ there is exactly one such path because $M$ is a DTM.

-Either the tape head is not scanning $\sigma_{J}(\mathrm{l})$, or $M$ is not in state $\mathrm{q}_{t}\left(\mathrm{q}_{2}\right)$

In the first case, $* *$ current_state is yes. Thus "**current_state $=$ \&not_valid" causes yes to point to not_vaid instead of valid_simulation. In the second case $* *($ tape_head $->\mathbf{s y m})$ is yes, and " $* *($ tape head->sym) = \&not_valid" causes yes to point to not_valid. The lemma is satisfied regardless of the subsequent code because yes points to not_valid.

We now proceed assuming that $M$ is scanning $\sigma_{l}$ (l) and is in state $\mathrm{q}_{z}\left(\mathrm{q}_{2}\right)$. Since $\delta\left(\mathrm{q}_{l}, \sigma_{J}\right)=\left(\mathrm{q}_{i}^{\prime}, \sigma_{j}^{\prime}, \mathrm{d}\right)$, we want to shift to state $\mathrm{q}_{l}^{\prime}$ ("current_state $=\& \mathrm{q}_{1}^{\prime}$ "), write $\sigma_{J}^{\prime}$ to the tape ("tape_head->sym $=\& \sigma_{J}^{\prime}$ "), and move the tape head

\footnotetext{
${ }^{4}$ We have not proven this here, but it follows from a simple inductive proof on the number of iterations of the while loop.
}

ACM Letters on Programming Languages and Systems, Vol. 1, No. 4, December 1992. 
one unit in direction $\mathrm{d}$ :

$$
\begin{aligned}
& \text { \#if } d=r \\
& \text { tape_head = tape_head }->\text { next; } \\
& \text { \#endif }
\end{aligned} \begin{gathered}
\text { \#if } d=L \\
\text { tape_head }=\text { tape_head }->\text { prev; } \\
\text { \#endif }
\end{gathered}
$$

Finally, the statements " $q_{i}=$ \&yes; $\sigma_{j}=$ \&yes" restore the condition that all state and alphabet variables point to yes. To finish our example, where $\delta\left(\mathrm{q}_{2}, \mathbf{1}\right)=\left(\mathrm{q}_{1}, \mathrm{e}, \mathrm{L}\right)$, the program store is now:

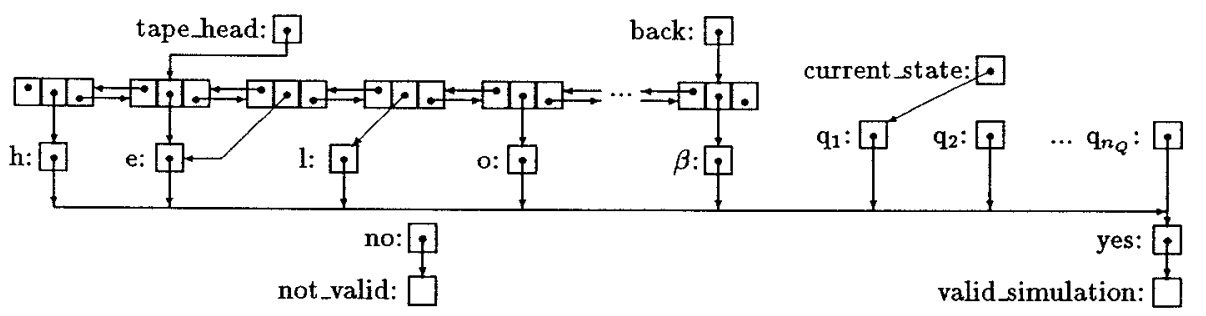

The rest of Figure 4 is addressed in the following lemma:

LEMMA 2.4. $M$ halts on $w$ iff on some path to $s$, in the program generated by reduce, ** current_state is valid_simulation.

Proof. Consider the program $C$ generated by reduce. By an inductive argument it is easy to show that:

(1) on all paths to the top of the while loop on which yes points to valid simulation, if the alias pattern represents $\mathrm{x}^{\prime} \mathrm{q}_{i}, \mathrm{y}^{\prime}$ then $\mathrm{q}_{0} \mathrm{w} \vdash{ }_{M}^{*} \mathrm{x}^{\prime} \mathbf{q}_{i}, \mathrm{y}^{\prime}$.

(2) if $\mathrm{q}_{0} \mathrm{w} \vdash{ }_{M}^{*} \mathrm{xq}$ y then there is at least one $\mathrm{path}^{5}$ to the top of the while loop generated by Figure 4 on which yes points to valid_simulation and on which the alias pattern represents $\mathrm{xq}_{i} \mathrm{y}$.

The proofs are by induction on the number of times the path has passed through the top of the loop and on the number of steps $M$ has taken to derive $\mathrm{xq}_{i} \mathrm{y}$. In both proofs, the base case is shown by Lemma 2.1, and the induction step can be shown by appealing to Lemmas 2.2 and 2.3. $M$ halts on w iff $\mathrm{q}_{0} \mathrm{w} \vdash{ }_{M}^{*} \mathrm{xq} \mathrm{f}_{f} \mathrm{y}$ (some $\mathrm{x}, \mathrm{y}$ ); thus $M$ halts on w iff there exists a path to the top of the loop on which yes points to valid_simulation and on which current_state points to $\mathrm{q}_{f}$.

Consider the effects of CHECK_ANSWER in Figure 4.

-If current_state does not point to $\mathbf{q}_{f}$, then $* *$ current_state must be not_valid.

-If yes points to not_valid, then $* *$ current_state must be not_valid.

-If current_state points to $q_{f}$ and if yes points to valid_simulation, then $* *$ current_state must be valid_simulation.

\footnotetext{
${ }^{5}$ Exactly one path unless $\mathrm{xq}_{L} \mathrm{y} \vdash \stackrel{+}{M} \mathrm{xq}_{L} \mathrm{y}$.
} 
This means that $M$ halts on $\mathrm{w}$ iff on some path to $s * *$ current_state is valid_simulation.

\section{MAY ALIAS IS NOT RECURSIVE}

THEOREM 3.1. Statically determining intraprocedural may alias for languages with if statements, loops, dynamic storage, and recursive data structures is recursively enumerable, but not recursive even when all paths through the program are executable.

Proof. Consider the program $C$ generated by reduce. All paths through $C$ are executable. Consider any path $P$; let $c_{1} c_{2} \ldots c_{k}$ be a sequence with a unique $c_{\imath}$ for every conditional (i.e., if and while statements) on $P$ in the order that they appear on $P$. Let $c_{\imath}$ be 1 if the true branch of the corresponding conditional is taken on $P$, and let $c_{t}$ be 0 otherwise. Clearly, $c_{1} c_{2} \ldots c_{k}$ is an input that executes path $P$. By Lemma $2.4, M$ halts on w iff on some path to $s$ in $C * *$ current_state is valid_simulation. Therefore, may alias is not recursive, since it can be used to solve the halting problem even for programs on which all paths are executable. May alias is recursively enumerable, since we can nondeterministically generate runs of a program and questions about aliasing during an execution can be answered by examining the symbol table and the program store.

THEOREM 3.2. Statically determining intraprocedure must alias for languages with if statements, loops, dynamic storage, and recursive data structures is not recursively enumerable even when all paths through the program are executable.

Proof. A quick look at the program produced by reduce (on which all paths are executable; see proof of Theorem 3.1) shows that yes either points to not_valid or valid_simulation; no always points to not_valid; $q \in$ $\mathrm{Q}$ always points to yes or no; and current_state always points to a state. This implies that $* *$ current_state is either valid_simulation or not_ valid. Since we already showed that $M$ halts on w iff on some path to $s$ ** current_state is valid_simulation (Lemma 2.4), it follows that $M$ does not halt iff $* *$ current_state is not_valid on all paths to $s$. Thus, must alias can be used to solve the complement of the halting problem that is not recursively enumerable. Thus must alias is not recursively enumerable given the language requirements stated by the theorem.

\section{CONCLUSION}

We have shown that intraprocedural may alias is not recursive and that intraprocedural must alias in not recursively enumerable even for programs on which all paths are executable, given that the language has dynamically allocated recursive data structures, loops, and if statements. This is an extremely negative result, considering that a doubly-linked list was the only dynamic data structure needed. Unfortunately, the proofs can be modified to 
use only a singly-linked list. In Appendix A we show how to modify reduce so that a singly-linked list is needed.

These results do not imply that the idea of static analysis is dead, but simply that, as has been known all along, some approximation must be done. What the new results do show is that, even if we are allowed to write exponential algorithms, static analysis would still have to be approximate. It probably also means that, in the presence of dynamically allocated recursive data structures, we have to accept approximations of lesser quality, which also take more time and space to compute than in Fortran. One final implication of our results is that while determining the structure of dynamic data structures (i.e., is it a tree? linked list?) is important, it is not sufficient to make static analysis recursive, because our proofs used only programs with linked lists.

Currently, static analysis is in the same situation as it was for Fortran 20 or so years ago. We are dealing with a problem that is not recursive, and we need to come up with a good set of simplifying assumptions and algorithms that yield reasonably good approximations quickly and cheaply. In most of the work in static analysis these assumptions are being introduced in an ad hoc manner, and are often not even explicitly stated. In the future it would be nice to have simplifying assumptions that would work for static analysis in general. One such assumption is that only polynomial-length paths need be considered. This seems reasonable, as we already assume that programs "terminate normally" (for example, no division by 0), and nonpolynomial executions are generally not tolerated. The nice result of this assumption is that it makes most static analyses $N P$ complete (see Appendix B). On the other hand, it is difficult to see how this would influence the development of approximate algorithms.

\section{Appendix A. Modification to Singly-Linked Lists}

The machine reduce (Figure 1) is modified to construct a program with only a singly-linked list and still satisfies Theorem 3.1 and Theorem 3.2, as follows:

- Remove prev field from struct tape. Also remove all statements dealing with prev.

- Add the field "int $* *$ not_at" to type struct tape. Point not_at to yes whenever a new tape element is created.

-Add a variable "struct tape $*$ front," which will always point to the first (leftmost) element of the linked list.

- Immediately before the while loop that simulates $\delta$, add the statement tape_head $->$ not_at $=$ \&no

In general, the not at field of the linked list representing the tape points to yes, except at tape_head where it points to no.

-Replace the code for when $d=R$ in the loop with

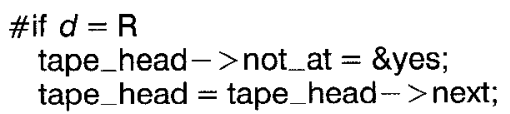


tape_head $->$ not_at $=$ \&no; \#endif

-Finally, replace the code for when $d=L$ in the loop with

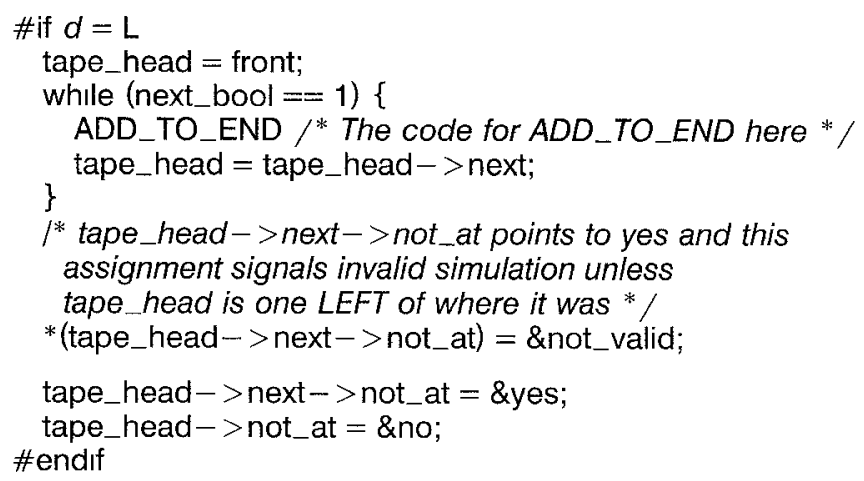

\section{Appendix B. Polynomial Paths Only}

When only polynomial-length paths are considered, may alias and the complement of must alias are NP complete for programs on which all paths are executable, given that the language has dynamically allocated recursive data structures, loops, and if statements. These problems are in NP because we can nondeterministically generate an execution of the program and then simulate the execution in time linear in the length of the execution (a similar argument is in [Larus 1989]). From the program store we can easily answer questions about may alias and the complement of must alias. This argument works for static-analysis problems that can be nondeterministically answered for a given execution in a polynomial amount of time, in the size of the program and length of the execution path, from the program, symbol table, and a possibly augmented program store. However, the augmented store must be polynomial in the size of the original store; for example, see Horowitz et al. [1989].

The fact that may alias and the complement of must alias are both NP hard under this new assumption is evident because our original proofs of this claim [Landi 1992; Landi and Ryder 1991] do not contain any loops and because only linear-length paths exist. Since most static analyses are influenced by aliases, they are also NP hard.

\section{ACKNOWLEDGMENTS}

We thank Rita Altucher, Bruce Ladendorf, Tom Marlowe, Michael Platoff, and the reviewers for their comments on this material.

\section{REFERENCES}

Aho, A. V. Hopcroft, J. E., ANd Ullman, J. D. 1976. The Design and Analysis of Computer Algorithms. Addison-Wesley, Reading, Mass.

Aho, A. V., Sethi, R., And Ullman, J. D. 1986. Compllers: Principles, Techniques, and Tools. Addison-Wesley, Reading, Mass.

ACM Letters on Programming Languages and Systems, Vol. 1, No 4, December 1992. 
Cousot, P., AND COUsot, R. 1977. Abstract interpretation: A unified lattice model for static analysis of programs by construction or approximation of fixpoints. In Conference Record of the 4th Annual ACM Symposium on Principles of Programming Languages. ACM, New York, $238-252$.

Hecht, M. S. 1977. Flow Analysis of Computer Programs. Elsevier North-Holland, New York. HopcrofT, J. E., AND Ullman, J. D. 1979. Introduction to Automata Theory, Languages, and Computation. Addison-Wesley, Reading, Mass.

Horwitz, S., Pfeiffere, P., AND Reps, T. 1989. Dependence analysis for pointer variables. In Proceedings of the ACM SIGPLAN Symposium on Compiler Construction. ACM, New York, $28-40$.

KaM, J. B., AND UlLman, J. D. 1977. Monotone data flow analysis frameworks. Acta Informatica 7, 305-317.

KAM, J. B., AND UlLMAN, J. D. 1976. Global flow analysis and iterative algorithms. J. ACM 23, $1,158-171$.

KILDALL, G. 1973. A unified approach to global program optimization. In Conference Record of the ACM Symposium on Principles of Programming Languages. ACM, New York, 194-206.

LANDI, W. 1992. Interprocedural aliasing in the presence of pointers. Ph.D. thesis, LCSR-TR174, Rutgers Univ., New Brunswick, N.J.

LANDI, W., AND RYDER, B. G. 1991. Pointer-induced aliasing: A problem classification. In Conference Record of the 18th Annual ACM Symposium on Principles of Programming Languages. ACM, New York, 93-103.

LARUS, J. R. 1989. Restructuring symbolic programs for concurrent execution on multiprocessors. Ph.D. thesis, Univ. of California, Berkeley.

LaRus, J. R., AND Hilfinger, P. N. 1988. Detecting conflicts between structure accesses. In Proceedings of the SIGPLAN '88 Conference on Programming Language Design and Implementation. ACM SIGPLAN Not. 23, 7, 21-34.

MYeRs, E. M. 1981. A precise interprocedural data flow algorithm. In Conference Record of the 8th Annual ACM Symposium on Principles of Programming Languages. ACM, New York, 219-230.

UlLman, J. D. 1973. Fast algorithms for the elimination of common subexpressions. Acta Informatica 2, 3, 191-213.

WEGMAN, M., AND ZADECK, F. K. 1991. Constant propagation with conditional branches. ACM Trans. Program. Lang. Syst. 13, 2 (Apr.), 181-210.

Received December 1992; revised March 1993; accepted May 1993 